\title{
Acute Care Surgery Model for Emergency Cholecystectomy
}

\author{
Myoung Je Song, M.D.*, Kyoung Mi Lee, M.D.*, In Byung Kim, M.D., Ph.D.*, \\ Heon-Kyun Ha, M.D. ${ }^{\dagger}$, Wan Sung Kim, M.D. ${ }^{\dagger}$, Hyoun Jong Moon, M.D., Ph.D. ${ }^{\dagger}$, \\ Jin Ho Jeong, M.D., Ph.D. ${ }^{\dagger}$, Kang Kook Choi, M.D., Ph.D. ${ }^{\ddagger}$ \\ Departments of *Emergency Medicine and ${ }^{\dagger}$ Surgery, Seonam University Myongji Hospital, Goyang, \\ ${ }^{\ddagger}$ Department of Trauma Surgery, Gachon University Gil Medical Center, Incheon, Korea
}

Correspondence to:

Kang Kook Choi, M.D., Ph.D. Department of Trauma Surgery, Gachon University Gil Medical Center, 21 Namdong-daero 774beon-gil, Namdong-gu, Incheon 21565, Korea

Tel: +82-32-460-3213

Fax: +82-32-461-3214

E-mail:

choikangkook@gilhospital.com
Purpose: Acute care surgery (ACS) models have evolved worldwide over the last decade. However, South Korea has an established trauma system and does not consider the ACS model. This study compares the management and outcome of emergency cholecystectomy in the ACS model to those of traditional on-call attending surgeon model for emergency surgery. Methods: Retrospectively collected data for patients who underwent emergency cholecystectomy from May 2013 to January 2015 was analyzed to compare data from a traditional on-call system (OCS) and ACS.

Results: One hundred and twenty-four patients were enrolled in the study (62 patients ACS vs. 62 patients $\mathrm{OCS}$ ). Hospital stay (days) $(\mathrm{ACS}=4.29 \pm 2.49$ vs. $\mathrm{OCS}=4.82 \pm 4.48, \mathrm{p}=0.46)$ and stay in emergency room (minutes) ( $\mathrm{ACS}=213.10 \pm 113.99 \mathrm{vs}$. $\mathrm{OCS}=241.10 \pm 150.73, \mathrm{p}=0.20$ ) did not differ significantly between groups. Operation time (minutes) was significantly shorter in the ACS than OCS group $(389.97 \pm 215.21$ vs. $566.35 \pm 290.14, \mathrm{p}<0.001)$. Other clinical variables (sex, open-conversion rate, whether the operation was performed at night/holiday, intensive care unit admission rate) did not differ between groups. There was no mortality and readmission. Conclusion: The implementation of the ACS led to shorter operation time and no increase of postoperative mortality and complication. (J Acute Care Surg 2016;6:57-61)

Key Words: General surgery, Time, Cholecystectomy, Gall bladder, Wounds and injuries

Received May 24, 2016, Revised June 16, 2016, Accepted June 16, 2016

*This article was presented at the 3rd Pan-Pacific Trauma Congress, June 4 6, 2015 in Seoul, Korea

Copyright (C) 2016 by Korean Society of Acute Care Surgery

(c) This is an Open Access article distributed under the terms of the Creative Commons Attribution Non-Commercial License (http://creativecommons.org/licenses/by-nc/4.0) which permits unrestricted non-commercial use, distribution, and reproduction in any medium, provided the original work is properly cited.

ISSN 2288-5862(Print), ISSN 2288-9582(Online)

https://doi.org/10.17479/jacs.2016.6.2.57

\section{Introduction}

Acute care surgery (ACS) model has evolved worldwide over the last decade. ACS is considered as a specific surgical service dedicated to the urgent assessment and treatment of patients with general surgical emergency including trauma [1,2]. Western countries have experienced fall of trauma surgery caused by development of imaging study, improved drug control, evolution of vascular intervention, and injury prevention [1,2].
ACS model has been suggested to overcome reduced operative management of blunt torso injury, increased financial burden, and challenge in maintaining surgical skill [1,2]. Now, ACS has become a standard model through a paradigm shift in western countries [1,2]. The trauma system of Korea is now being established under the leadership of the public health authority; however, the ACS model has not been adopted.

Acute cholecystitis is one of the most common surgical problems in need of emergency surgery [3]. The prognosis of 
acute cholecystitis varies widely from uneventful cure after cholecystectomy to lethal complication like biliary sepsis [4,5]. Early cholecystectomy can reduce the complication rate and hospital stay of acute cholecystitis patients $[4,5]$.

Even though ACS model was successfully implemented in many hospitals worldwide, relatively few studies have focused on the effect of ACS for the non-trauma surgical emergency $[3,6,7]$. The previous studies showed that ACS model improved work flow in emergency department, decreased surgical evaluation time and hospital length of stay, as compared to traditional on-call system (OCS) [3,8-10]. The aim of this study was to elucidate the impact of the ACS model to emergency cholecystectomy by comparing the management and outcome of emergency cholecystectomy in ACS model to those of traditional on-call attending surgeon model for emergency surgery.

\section{Methods}

A retrospective analysis of patients who underwent emergency cholecystectomy in the hospital was performed to com- pare data from traditional OCS and ACS from May 2013 to January 2015. Each protocol was reviewed and approved by the institutional review board of Myongji Hospital. In this period, ACS model was partially implemented in the hospital. The ACS model and OCS model were applied alternatively for the study period for the emergency surgery.

Exclusion criteria of the study were the following: patients with 1) preoperative/postoperative endoscopic retrograde cholangiopancreatography (ERCP); 2) accompanied choledocholithiasis, cholangitis; 3) accompanied procedure including choledocholithotomy, choledochostomy or other combined surgical procedure; 4) sepsis needed to resuscitation; 5) intra- abdominal adhesion due to previous operation or medical condition; 6) severe combined pancreatitis; 7) other medical conditions that needed further diagnosis or additional treatment including pneumonia, heart failure; 8) single incision laparoscopic cholecystectomy; and 9) immediate transfer to other hospital after cholecystectomy (Fig. 1).

The emergency cholecystectomy was performed by 2 attending surgeons dedicated to emergency surgery including trauma in

Exclusion criteria: total 115 patients

1) Preoperative/postoperative ERCP: 14 patients

2) Accompanied choledocholithiasis, cholangitis: 11 patients

3) Accompanied procedure including choledocholithotomy, choledochostomy or other combined surgical procedure: 15 patients

4) Sepsis needed to resuscitation: 14 patients

5) Intra-abdominal adhesion due to previous operation or medical condition: 6 patients

6) Severe combined pancreatitis: 7 patients

7) Other medical condition need to further diagnosis or additional treatment including pneumonia, heart failure: 30 patients

8) Single incision laparoscopic cholecystectomy: 13 patients

9) Immediate transfer to other hospital: 5 patients

A total 239 cholecystectomy was performed in the study period.
A total 124 patients were erolled in this study.
Fig. 1. Exclusion criteria of the study. ERCP: endoscopic retrograde cholangiopancreatography. 
ACS group and by 3 other conventional on-call surgeons in OCS group. A retrospective analysis was performed for the basic patient characteristics and clinical factors including sex, age, operation method, open conversion, whether the surgery was performed on holiday (weekend and other national holiday), whether the surgery was performed at night (6 PM $\sim 7 \mathrm{AM})$, intensive care unit (ICU) admission, emergency room (ER) stay, time to operation, operation time, and hospital stay.

All continuous data were presented as mean \pm standard deviation, unless otherwise noted. $\chi^{2}$ test was used for nominal and categorical variables and the t-test was used for continuous variables. A p-value $<0.05$ (two tailed) was considered statistically significant. All statistical analyses were performed with IBM SPSS Statistics ver. 19.0 for Windows (IBM Co., Armonk, NY, USA).

\section{Results}

A total 239 emergency cholecystectomies were performed in this period. Finally, 124 patients were enrolled after 115 exclusions according to the exclusion criteria of the study (Fig. 1). There was no mortality and readmission. ACS group was younger than OCS group $(57.66 \pm 15.97$ vs. $48.56 \pm 14.73, \mathrm{p}<$ 0.01; Table 1). Hospital stay (d) and stay in ER (min) was not significantly different between OCS and ACS group (4.29 2.49 vs. $4.82 \pm 4.48, \mathrm{p}=0.46 ; 213.10 \pm 113.99$ vs. $241.10 \pm 150.73$, $\mathrm{p}=0.20$; Table 1 ). Time to operation ( $\mathrm{min}$ ) was significantly shorter in ACS than OCS group (389.97 \pm 215.21 vs. $566.35 \pm$ 290.14, p <0.001; Table 1). Laparoscopic-cholecystectomy rate of ACS group was higher than that of OCS group, with no statistical significance ( $3.2 \%$ vs. $11.3 \%, p=0.05$; Table 1 ). Other clinical variables (sex, open-conversion rate, whether the operation was performed at night/holiday, and ICU admission rate) did not show differences between the two groups (Table 1). Post-operative complication occurred as follows: 1 patient of OCS group had pneumonia after the operation; 1 patient had wound seroma of umbilical port site in each group individually.

\section{Discussion}

The purpose of this study was to determine the impact of ACS model to emergency cholecystectomy. There has been no such study for impact of ACS in Korea. We evaluated the clinical impact of ACS in emergency surgery especially in cholecystectomy because cholecystectomy is one of the most common emergency surgical procedures. Appendectomy is also a common emergency procedure, but since it is technically easier compared to cholecystectomy it is often performed by in-house residents. This can cause bias when comparing outcomes such as time to operation between groups.

The five studies were performed to determine the impact

Table 1. Demographics, work flow and outcomes between groups $(n=124)$

\begin{tabular}{lccr}
\hline & Acute care surgery group (n=62) & On-call system group (n=62) & p-value \\
\hline Sex (male/female) & $30 / 32$ & $33 / 29$ & 0.72 \\
Age (y) & $57.66 \pm 15.97$ & $48.56 \pm 14.73$ & $<0.01$ \\
Open cholecystectomy & $2(3.2)$ & $7(11.3)$ & 0.05 \\
Open conversion & 0 & $1(1.6)$ & 1.00 \\
Holiday surgery & $18(29.0)$ & $19(30.6)$ & 1.00 \\
Night surgery & $33(53.2)$ & $32(51.6)$ & 1.00 \\
Holiday or night surgery & $24(38.7)$ & $20(32.3)$ & 0.57 \\
Intensive care unit admission & $8(12.9)$ & $1(1.6)$ & 0.34 \\
Emergency room stay (min) & $213.10 \pm 113.99$ & $241.10 \pm 150.73$ & 0.20 \\
Time to operation (min) & $389.97 \pm 215.21$ & $566.35 \pm 290.14$ & $<0.001$ \\
Operation time (min) & $73.79 \pm 31.44$ & $63.39 \pm 36.46$ & 0.09 \\
Hospital stay (d) & $4.29 \pm 2.49$ & $4.82 \pm 4.48$ & 0.46 \\
\hline
\end{tabular}

Values are presented as number only, mean \pm standard deviation, or number (\%). 
Table 2. The impact of ACS model compared to traditional OCS model in previous studies

\begin{tabular}{lllll}
\hline \multicolumn{1}{c}{ Author } & Time to operation & Open conversion & Hospital stay & Mortality or morbidity \\
\hline Lehane et al. [9] & Shorter & NS & Less & Less \\
Britt et al. [8] & Shorter & NS & NS & NS \\
Lau and Difronzo [3] & Shorter & NS & Less & Less \\
Lim et al. [7] & Shorter & NS & Less & NS \\
Michailidou et al. [11] & Shorter & Less & NS & Less \\
The current study & Shorter & NS & Less \\
\hline
\end{tabular}

ACS: acute care surgery, OCS: on-call system, NS: non-significant.

of ACS implementation on biliary disease (Table 2) [3,7-9,11].

The two studies included only acute cholecystitis [3,9] and the others included biliary tract disease widely $[7,8,11]$. However, the study designs were essentially similar to each other in the comparison between ACS and OCS in biliary tract disease/ cholecystitis. In all previous studies, time to operation of ACS was shorter than that of OCS. It may be caused by faster decision making and early intervention of in-house staff in ACS group than those of OCS group. Contrarily, conversion to open surgery rate was significantly less only in one study [11] than that of OCS. The three studies in which the ACS group had less hospital stay than that of OCS group, showed less complication rate in ACS group than OCS group [3,9,11]. However, ACS group did not increase postoperative complication, as compared to the OCS group in any previous studies [3,7-9,11]. Only two studies elucidated the difference of cost between groups with significant decrease of the cost in ACS group compared to OCS group [8,11].

ACS model have several benefits due to the following: 1) Trauma surgeons as in-hospital staff are always under readiness for action; 2) Trauma surgeons are relatively more comfortable in treating critically ill patients than conventional surgeons; 3) ACS model can reduce financial/human burden by sharing resources; 4) Above all, ACS model is necessary for maintaining surgical skills of trauma surgeon by increasing the surgical opportunities; and 5) Additionally, patients who needed emergent surgery other than trauma are also important. ACS model can provide consistent and timely coverage.

The current study has mainly two limitations. The first is from the retrospective study design. The second concerns the surgeon factor. The result can vary widely between hospitals based on skill and policy of the attending surgeon. While our results showed faster time to operation of ACS group than OCS group, the difference was not sufficient to change clinical outcome. The OCS surgeons of our hospital did not have their own protocol for acute cholecystitis, but they have a policy by which they do not delay the operation without a specific reason.

In conclusion, implementation of the ACS showed the shorter time to operation and no increase of postoperative mortality and complication. The true effect of ACS should be evaluated in terms of clinical, educational, and financial aspects. The United States has recognized the need to implement ACS because of the limitation of trauma system through many trials and error [12]. Korea is now establishing the trauma system and can take a lesson from the good role model, which has developed the trauma system. We believe that the public health authority of Korea should consider the implementation of ACS model.

\section{Conflicts of Interest}

No potential conflict of interest relevant to this article was reported.

\section{References}

1. Velmahos GC, Jurkovich GJ. The concept of acute care surgery: a vision for the not-so-distant future. Surgery 2007;141: 288-90.

2. Ball CG, Hameed SM, Brenneman FD. Acute care surgery: a new strategy for the general surgery patients left behind. Can J Surg 2010;53:84-5.

3. Lau B, Difronzo LA. An acute care surgery model improves 
timeliness of care and reduces hospital stay for patients with acute cholecystitis. Am Surg 2011;77:1318-21.

4. Casillas RA, Yegiyants S, Collins JC. Early laparoscopic cholecystectomy is the preferred management of acute cholecystitis. Arch Surg 2008;143:533-7.

5. Siddiqui T, MacDonald A, Chong PS, Jenkins JT. Early versus delayed laparoscopic cholecystectomy for acute cholecystitis: a meta-analysis of randomized clinical trials. Am J Surg 2008; 195:40-7.

6. Cubas RF, Gómez NR, Rodriguez S, Wanis M, Sivanandam A, Garberoglio CA. Outcomes in the management of appendicitis and cholecystitis in the setting of a new acute care surgery service model: impact on timing and cost. J Am Coll Surg 2012;215:715-21.

7. Lim DW, Ozegovic D, Khadaroo RG, Widder S. Impact of an acute care surgery model with a dedicated daytime operating room on outcomes and timeliness of care in patients with biliary tract disease. World J Surg 2013;37:2266-72.
8. Britt RC, Bouchard C, Weireter LJ, Britt LD. Impact of acute care surgery on biliary disease. J Am Coll Surg 2010;210:595-9, 599-601.

9. Lehane CW, Jootun RN, Bennett M, Wong S, Truskett P. Does an acute care surgical model improve the management and outcome of acute cholecystitis? ANZ J Surg 2010;80: 438-42.

10. Qureshi A, Smith A, Wright F, Brenneman F, Rizoli S, Hsieh $\mathrm{T}$, et al. The impact of an acute care emergency surgical service on timely surgical decision-making and emergency department overcrowding. J Am Coll Surg 2011;213:284-93.

11. Michailidou M, Kulvatunyou N, Friese RS, Gries L, Green DJ, Joseph B, et al. Time and cost analysis of gallbladder surgery under the acute care surgery model. J Trauma Acute Care Surg 2014;76:710-4.

12. Coleman JJ, Esposito TJ, Rozycki GS, Feliciano DV. Acute care surgery: now that we have built it, will they come? J Trauma Acute Care Surg 2013;74:463-8; discussion 468-9. 